\title{
Effect of Ticagrerol versus Clopidogrel on Oxidative Stress Bio-markers in Patients with Chronic Stable Angina after Percutaneous Coronary Intervention
}

\author{
ELORA SHARMIN ${ }^{1}$, JASMINE FAUZIA DEWAN ${ }^{2}$, SYED ALI AHSAN ${ }^{1}$, HARISUL HOQUE ${ }^{1}$, SHEIKH FOYEZ \\ AHMED $^{1}$, MD. ASHRAF UDDIN SULTAN ${ }^{1}$, HABIBAAKHTER BHUIYAN ${ }^{2}$ SHIMA BHADRA ${ }^{1}$, CHAITY BARUA ${ }^{1}$ \\ ${ }^{1}$ Department of Cardiology,Bangabandhu Sheikh Mujib Medical University, Dhaka, ${ }^{2}$ Department of Pharmacology, Bangabandhu \\ Sheikh Mujib Medical University, Dhaka \\ Address for Correspondence: Dr. Elora Sharmin, Medical officer, Department of Cardiology, Bangabandhu Sheikh Mujib \\ Medical University, Dhaka. Email:eloradoc@gmail.com
}

\begin{abstract}
Background: Ischemic heart disease remains the leading cause of death in both developed and under developed countries. The use of antiplatelet drugs specifically the thienopyridine has become a standard for the treatment of acute coronary syndrome. These drugs irreversibly inhibit the platelet aggregation by blocking the P2Y12 receptor. But currently this therapeutic choice has become limited due to potential interaction with other drugs, slow hepatic conversion, genetic resistance and narrow therapeutic safety margin. Ticagrerol, a reversible P2Y12 receptor inhibitor may represent a significant advancement over currently available oral antiplatelet drugs. Objectives: The study was intended to compare the effect of Ticagrelor and Clopidogrel on oxidative stress markers in patients of chronic stable angina (CSA) following percutaneous coronary intervention (PCI). Materials \& Methods: The present prospective observational study was carried out in the Department of Pharmacology, Cardiology and Microbiology, BSMMU, Dhaka from September 2014 to February 2016. The study included a total of 100 CSA patients. Patients were divided into two groups, Ticagrelor and Clopidogrel treated groups (each having 50 patients). The baseline laboratory parameters-Malondihyde (MDA), Reduced glutathione (GSH), bleeding time, clotting time and platelet count, were measured and then patients of both groups underwent PCI. The same parameters were again assessed at follow up after 4 weeks of intervention. Total 12 patients from Ticagrelor and 14 patients from Clopidogrel groups were dropped out. Comparisons of the laboratory parameters were made between two groups at baseline and at follow up and also within group before and after intervention. Result: In the present study at baseline characteristics of patients treated with ticagrelor and clopidogrel were almost identical in terms of age, sex, diabetes and hypertension. The level of plasma MDA in ticagrelor group was significantly reduced from baseline to follow up $(4.5 \pm 1.8$ to $1.4 \pm 0.7$, $p<0.001)$ and in clopidogrel group $(4.2 \pm 1.2$ to $1.3 \pm 0.7, p<0.001)$. GSH level was increased from $0.7 \mathrm{mg} / \mathrm{dl}$ to $2.5 \mathrm{mg} / \mathrm{dl}(\mathrm{p}<0.001)$ in ticagrerol group and in clopidogrel group $0.6 \mathrm{mg} / \mathrm{dl}$ to $1.4 \mathrm{mg} / \mathrm{dl}, \mathrm{p}<0.001)$. Conclusion: The study concluded that both ticagrelor and clopidogrel are similar effect on oxidative stress markers, resulting from oxidative injury processes in patients of chronic stable angina.
\end{abstract}

Introduction:

Coronary heart disease is a major global health problem in European country ${ }^{1}$ Low and middle income countries, including South Asian countries like India and Pakistan, contribute significantly to the global burden of cardiovascular disease. A projection made by Murray and Lopez (1997) showed that by 2020, 78\% of all deaths and $86.3 \%$ of all loss of disability adjusted life years (DALYs) will be attributable to this cause ${ }^{2}$. The malondialdehyde and reduced glutathione are sensitive markers of oxidative stress $^{3}$. In normal cell metabolism, free radicals are generated by biochemical redox reactions. Pathogenesis of a variety of vascular diseases including atherosclerosis, hypertension and coronary artery disease are implicated by reactive oxygen species (ROS) which are produced from oxidative stress ${ }^{4}$. In coronary artery disease there is alteration in oxidant-antioxidant profile. In coronary artery disease, increased secretion of TNF- \pm which contributes to depression of extracellular SOD activity ${ }^{5}$.This leads to endothelial dysfunction in coronary artery disease by increasing free radical load and inactivation of NO by superoxide anions forming peroxynitite ${ }^{5}$.

In Pharmacology, anti-oxidative function of drugs is measured by assessing presence or absence of oxidative markers respectively.

Ticagrelor, a comparatively recently introduced ADPreceptor inhibitor, is a member of the theienopyridine class of adenosine diphosphate (ADP) receptor inhibitors which reduces platelet aggregation by reversibly binding to ADP 
receptors on platelet membrane ${ }^{6}$. Compared to clopidogrel and prasugrel, ticagrelor inhibit adenosine diphosphate (ADP) induced platelet aggregation more rapidly and more consistently to a greater extent both in the healthy subjects and in patients with coronary artery disease including those undergoing percutaneous coronary intervention (PCI ${ }^{7}$ and reduce the risk of death either resulting from vascular cause, myocardial infraction (MI) or stroke ${ }^{8}$. For this reversible binding of ticagrelor to ADP-receptors, the post percutaneous bleeding tendency in CSA patients would expectedly be lower than that in patients receiving clopidogrel ${ }^{9}$.

In patients of ACS following $\mathrm{PCI}$, the chance of thrombotic phenomenon is increased because of oxidative injury .In that situation an antiplatelet drug having anti- oxidative property could be a better option. Therefore the present study has been designed to compare the anti-oxidative properties of ticagrelor and clopidogrel in CSA patients following PCI.

\section{Materials and Methods:}

The present prospective observational study was carried out in the Department of Pharmacology, Cardiology and Microbiology, BSMMU, Dhaka from September 2014 to February 2016. Patients who were to suffer from as a case of chronic stable angina and under-going PCI in cardiology department of BSMMU and also fulfilled theexclusion criteria were the study population.

\section{Inclusion criteria-}

i) Age: between 18 to $75 \mathrm{yrs}$.

ii) Sex: Both

iii) High sensitive C-reactive protein (hs- CRP) level e" $3.0 \mathrm{mg} / \mathrm{L}$ (Morrow et al., 1998)

iv) Patients who gave written informed consent to participate in the study.

\section{Exclusion criteria:}

i) Patients who were diagnosed as STEMI, NSTEMI

ii) Patients receiving one or more dose of theinopyridine for 5 days or less before PCI

Patients were divided into two groups, Ticagrelor $(90 \mathrm{mg})$ plus aspirin $(75 \mathrm{mg}$ ) and Clopidogrel $(150 \mathrm{mg})$ plus aspirin $(75 \mathrm{mg})$ treated groups, each having 50 patients. In laboratory malondialdehyde (MDA), reduced glutathione (GSH), bleeding time, clotting time, were measured and then patients of both groups underwent PCI. The same parameters were again assessed at follow up after 4 weeks of intervention. Comparisons of the laboratory parameters were made between two groups at baseline and at follow up and also within group before and after intervention.

\section{Estimation of plasma malondialdehyde (MDA) level ${ }^{10}$}

Lipid peroxidation, defined as the oxidative deterioration of polyunsaturated fatty acids, is a free radical induced phenomenon. Lipid peroxides are unstable and decompose to form a complex series of compounds including reactive carbonyl compounds. Polyunsaturated fatty acids peroxides generate malondialdehyde (MDA) upon decomposition. Many years ago, a simple and reliable method for the assay of lipid peroxides level in serum or plasma by use of the thiobarbituric acid reaction was advised. The measurement of thiobarbituric acid reactive substances (TBARS) is a well-established method for screening and monitoring lipid peroxidation. Even though there remains a controversy cited in literature regarding the specificity of TBARS towards compounds other than MDA, it still remains the most widely employed assay used to determine lipid peroxidation.

\section{Estimation of erythrocyte glutathione (GSH) level by Ellman's method ${ }^{11}$}

The simple spectrophotometric procedure for GSH estimation is based on the method of Ellman, who reported that 5,5-dithiobis-2-nitrobenzoic acid is reduced by $\mathrm{SH}$ group to form 1 mole of 2-nitro-5-mercaptobenzoic acid per mole of (-SH). The nitromercaptobenzoic acid anion has an intense yellow color and can be used to measure SH groups. The optimal condition for color development and preparation of homogenates has been studied with respect to precision, reducibility and specificity of the estimation 17. This color intensity was measured by the spectrophotometer (UV-VIS spectrophotometer) at $412 \mathrm{~nm}$ wavelength.

\section{Result:}

The present study aimed at comparing the effect of Ticagrelor and Clopidogrel on oxidative stress markers in patients of chronic stable angina (CSA) following PCI. The baseline laboratory parameters (MDA, GSH, bleeding time, clotting time and platelet count) were measured and then both groups underwent PCI. The same parameters were again assessed at follow up after 4 weeks of PCI discharged with ticagrelor and clopidogrel. Total 12 patients from Ticagrelor and 14 patients from Clopidogrel groups dropped out leaving 38 in the former and 36 in the latter group for final analysis. Comparisons of the laboratory parameters were made between groups both at baseline and at follow up and within group before and after intervention. The findings of the study derived from data analyses are presented below: 


\section{Baseline characteristics}

Table-shows the distribution of baseline characteristics between Ticagrelor and Clopidogrel groups

There was no significant difference between the groups in terms of age with mean age of the patients being around 50 years $(\mathrm{p}=0.322)$. The sex distribution was identical with male predominance in either group $(p=0.328)$. About one-quarter $(22.5 \%)$ of the Ticagrelor group and one-third $(32.5 \%)$ of the Clopidogrel group were diabetic $(p=0.201)$. Over two-thirds (70\%) of the former and $65 \%$ of the latter group had hypertension with no significant intergroup difference $(\mathrm{p}=0.208)$.

Baseline characteristics of Ticagrerol and Clopidogrel groups

\begin{tabular}{lccc}
\hline $\begin{array}{l}\text { Baseline } \\
\text { characteristics }\end{array}$ & $\begin{array}{c}c \\
\text { Ticagrerol } \\
(\mathrm{n}=38)\end{array}$ & $\begin{array}{c}\text { Clopidogrel } \\
(\mathrm{n}=36)\end{array}$ & \\
\hline Age (years) & $50.7 \pm 8.5$ & $48.9 \pm 7.3$ & $0.332^{*}$ \\
Sex & & & \\
Male & $30(75.0)$ & $33(82.5)$ & \\
Female & $10(25.0)$ & $7(17.5)$ & $0.328^{\mathrm{D}}$ \\
Diabetes mellitus & $9(22.5)$ & $13(32.5)$ & $0.201^{\mathrm{D}}$ \\
Hypertension & $28(70.0)$ & $26(65.0)$ & $0.208^{\mathrm{D}}$ \\
\hline
\end{tabular}

Figures in the parentheses indicate corresponding \%; ${ }^{{ }}$Chi-squared Test $\left(\mathrm{c}^{2}\right)$ was done to analyze the data.

*Data were analyzed using unpaired t-Test and were presented as mean $\pm \mathrm{SD}$.

tiarerol

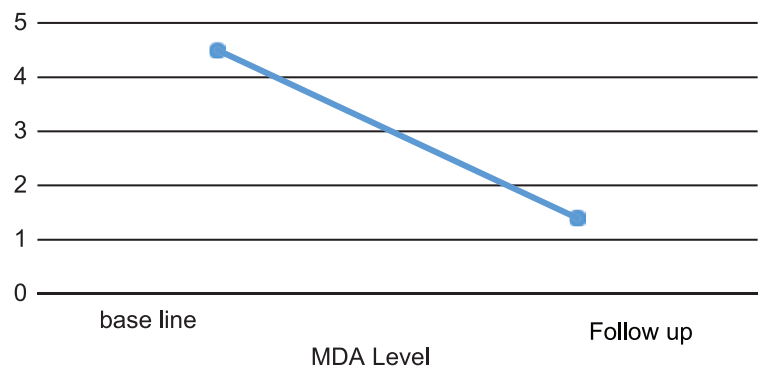

ticagreol

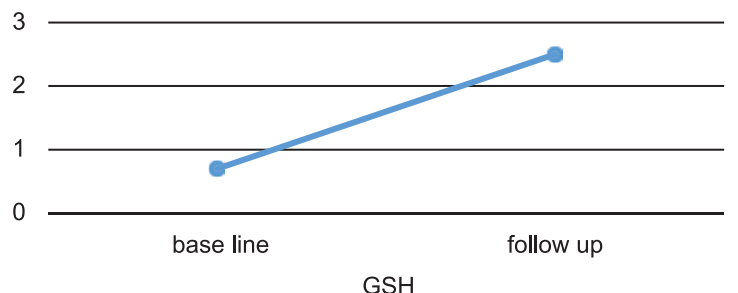

GSH
Lab findings of MDA level in both groups at baseline and follow up

The mean plasma MDA levels of Ticagrelor and Clopidogrel groups were almost similar $\left(4.5 \pm 1.8^{1 / 4 \mathrm{~mol} / \mathrm{L}}\right.$ vs. $\left.4.2 \pm 1.2 \frac{1}{4} \mathrm{~mol} / \mathrm{L}, \mathrm{p}=0.306\right)$. The level of plasma MDA in both Ticagrelor and Clopidogrel groups were decreased at follow up 4 weeks after PCI $(1.4 \pm 0.7$ vs. $1.3 \pm 0.7,1 / 4 \mathrm{~mol} /$ $\mathrm{LP}=0.580$ ). Data were analyzed using unpaired $\mathrm{t}$-Test and were presented as mean $\pm \mathrm{SD}$

\section{Lab findings of erythrocyte GSHlevel in both groups at baseline and follow up}

The erythrocyte GSH was also identically distributed between groups $(p=0.958)$. The level of GSH was increased in both groups with increase being significantly more in ticagrerol group than clopidogrel ( $2.5 \pm 0.9$ vs. $1.4 \pm 0.8 \mathrm{mg}$ / gm of $\mathrm{Hb}, \mathrm{p}<0.001$ ). Data were analyzed using unpaired tTest and were presented as mean $\pm \mathrm{SD}$

Percentage of changes in oxidative stress markers (GSH and MDA) in Ticagrerol and Clopidogrel group

GSH increase from baseline to follow up in Ticagrelor and Clopidogrel groups were 257.1 and 133.3 respectively and the difference was statistically significant. (0.005). Percentage of decrease in MDA was almost similar in both Ticgrelor and Clopidogrel group (68.8\% vs. $69.0 \%$, $\mathrm{p}=0.435)$.
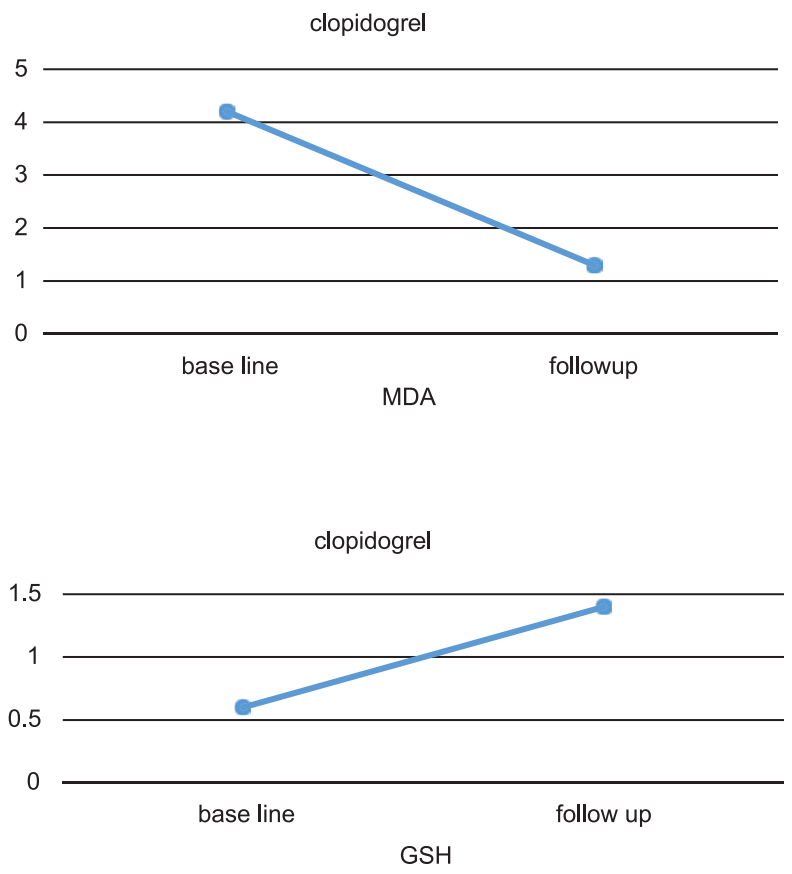


\begin{tabular}{|c|c|c|c|c|c|c|c|c|}
\hline \multirow[t]{2}{*}{ 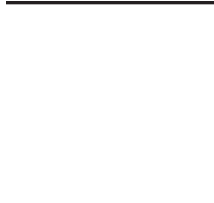 } & \multirow[t]{2}{*}{ Markers } & \multicolumn{3}{|c|}{ Ticagrelor group } & \multicolumn{3}{|c|}{ Clopidogrel group } & \multirow[b]{2}{*}{$\begin{array}{l}\mathrm{P} \text { value } \\
\downarrow\end{array}$} \\
\hline & & $\begin{array}{c}\text { Baseline } \\
\text { Mean } \pm \text { SD }\end{array}$ & $\begin{array}{c}\text { After } 4 \text { weeks } \\
\text { Mean } \pm \text { SD }\end{array}$ & $\begin{array}{c}\% \text { of } \\
\text { Increased } \uparrow \\
\text { or } \\
\text { decreased } \downarrow\end{array}$ & $\begin{array}{c}\text { Baseline } \\
\text { Mean } \pm \text { SD }\end{array}$ & $\begin{array}{c}\text { After } 4 \text { weeks } \\
\text { Mean } \pm \mathrm{SD}\end{array}$ & $\begin{array}{c}\% \text { of } \\
\text { Increased } \uparrow \\
\text { or } \\
\text { decreased } \downarrow\end{array}$ & \\
\hline Oxidative stress & $\mathrm{GSH}(\mathrm{mg} / \mathrm{gm}$ of $\mathrm{Hb})$ & 0.7 & 2.5 & $257.1 \uparrow$ & 0.6 & 1.4 & $133.3 \uparrow$ & 0.005 \\
\hline markers & $\operatorname{MDA}(1 / 4 \mathrm{~mol} / \mathrm{L})$ & 4.5 & 1.4 & $68.8 \downarrow$ & 4.2 & 1.3 & $69.0 \downarrow$ & 0.435 \\
\hline
\end{tabular}

\section{Discussion}

Coronary artery disease (CAD) is the major cause of mortality and morbidity worldwide ${ }^{12,13}$. Blockade of blood flow inside the coronary arteries or narrowing of the arterial lumina due to atherosclerotic changes are the primary factors leading to CAD. Free radicals are formed in both physiological and pathological conditions in mammalian tissues including cardiac tissues ${ }^{14}$. Alteration in the oxidant-antioxidant profile may occur in many diseases including $\mathrm{CAD}$. If not nullified by endogenous antioxidants of the body or if endogenous anti-oxidant become depleted due to any cause, uncontrolled elevation of free radicals may occur and may lead to tissue damage or tissue necrosis. Previous studies have suggested that oxidative stress may be involved in $\mathrm{CAD}$ and that higher production of free radical production and decreased anti-oxidant activity are indicative of oxidative stress in $\mathrm{CAD}^{3}$.

In the present study two PCI groups receiving ticagrelor and clopidogrel along with aspirin were taken for comparison between the outcomes of either drug administration following PCI. The objective was to assess whether clopidogrel or ticagrerol would provide better results if administered in CAD patients following PCI. The patients groups taken were almost identical in terms of their demographic characteristics (age and sex). Disease conditions such as diabetes and hypertension were equally distributed between the groups. The parameters to be measured in the present study such as MDA, erythrocyte GSH, platelet count, bleeding time and clotting time when obtained prior to drug administration appeared similarly distributed between the treatment groups. Thus as most of the baseline characteristics were similar in distribution between the comparative groups, the outcome obtained could be assumed to be due to intervention by the administered drugs.

The oxidant-antioxidant markers the MDA (product of lipid peroxidation indicative of oxidant stress) levels were high in either ticagrerol or clopidogrel group prior to intervention by the drugs. This finding is in accordance with that of ${ }^{12}$, who have stated that patients of CAD show increased oxidative stress. Stressful conditions lead to oxidant-antioxidant imbalance and higher lipid peroxidation. The higher the lipid peroxidation, the higher becomes the level of MDA in tissues. After ticagrerol or clopidogrel administration, both groups demonstrated reduced levels of MDA. This would suggest that the process of lipid peroxidation was reduced following clopidogrel and ticagrerol administration, as a result of reduced oxidative stress. The pattern of reduction of MDA levels were similar in both groups. A reason might be that the MDA values obtained from both groups prior to drug administration were of similar pattern perhaps due to identical demographic characters and, perhaps the identical demographic characteristic of both drug- treated groups were responsible for these findings. However, results of the present study indicate that both ticagrerol and clopidogrel were almost equal effective in exerting anti-lipid peroxidation effect. These observations of the present study resemble to those of ${ }^{3,12}$.

The reduced glutathione (GSH) levels in $\mathrm{RBC}$ membranes of both groups before drug administration were lower $(0.7$ \pm 0.3 in ticagrerol group and $0.6 \pm 0.2$ in clopidogrel group). This would suggest that glutathione levels become depleted in CAD patients perhaps due to oxidants produced as a result of stress and are similar findings ${ }^{12}$. We propose that the decreased levels of the anti-oxidants may be a compensatory regulation in response to increased oxidative stress as has also been suggested ${ }^{3}$. Significant elevations have occurred in GSH levels in both groups following treatment by either clopidogrel or ticagrerol after 4 weeks of administration of the drugs ( $2.5 \pm 0.9$ in ticagrerol group and to $1.4 \pm 0.8$ in the clopidogrel group, $\mathrm{p}<0.001$ ). This would suggest that administration of both drugs in either group could replenish the GSH levels. This finding is similar ${ }^{3,12}$ and support their findings that the levels of antioxidant enzymes remains lower in $\mathrm{CAD}$ patients, which may become replenished when recovery occurs. In the present study, the replenishment of GSH was more evident in the ticagrerol treated group of patients, suggesting that the anti-oxidant and glutathione replenishing efficacy of ticagrerol was more obvious compared to that of clopidogrel. 


\section{Conclusion:}

From the findings of the present study, it appears that both ticagrelor and clopidogrel are effective in improving the status of oxidative stress markers resulting from oxidative injury caused by chronic stable angina. But in terms of outcome, ticagrelor could be considered better than the clopidogrel.

\section{References:}

1. Levi, F, Lucchini,F, Negri, E, and La, Veenchia, C, Trends in mortality from cardiovascular and cerebrovascular disease in Europe and other areas of the world.Heart British Medical Journal, 2002;88(2):119-24.

2. Murray, C. J, Lopez, A.D, Mortality by cause for eight regions of the world: Global Burden of Disease study.Lancet, 2001; 349:1269-76.

3. Aparna, P, Betigeri, M. A, Pasupathi, P, Homocysteine and oxidative stress markers and inflammation in patients with coronary artery disease. International Journal of Biological and Medical Research, 2010;1(4):125- 29.

4. Muzakova, V, Kandar, R, Vojtisek, P, Shalicky, Vankova, R, Cegan, A, Cervinkova, Z, antioxidant vitamin level and glutathione peroxidase activity during ischemia/ reperfusion in myocardial infraction. Physiology research, 2001; 50(4):389-396.

5. Ulf, L, Merten, R, Spiekermann, S, Buttner, K, Drexler, H, Hornig, B, Vascular extracellular superoxide dismutase activity in patients with coronary artery disease. Circulation, 2000;101:2264-79.
6. Husted, S, Van Giezen, J.J, Ticagrelor: the first reversibly binding oral P2Y12 receptor antagonist. Cardiovascular Therapeutics, 2009;27(4):259-74.

7. Birkeland, K, Parra, D, Rosenstein, R. Antiplatelet therapy in acute coronary syndromes: focus on ticagrelor. Journal of Blood Medicine, 2010;1:197-219.

8. Showkathali, R, Natarajan, A, Antiplatelet and Antithrombin Strategies in Acute Coronary Syndrome: State-Of-The-Art Review.Current Cardiology Revised, 2012;8(3):239-249.

9. Pepys, M.B, Williams, G, C-reactive protein and amyloidosis from protein to drug. In: Royal college of physicians of London. 1999; p. 397-414.

10. Yagi, K. 'Lipid peroxides and human diseases' Chemistry Physics Lipids, 1978;45:337-371.

11. Ellman, G .L.1959, 'Tissue sulfhydryl groups', Archive Biochemistry Biophysics, 1959;82:70-77.

12. Kaur, K., Bedi, G., Kaur, M., Anil, V., Kaur, I. 'Lipid Peroxidation and the level of Antioxidant Enzymes in Coronary Artery Disease', Indian Journal of Clinical Biochemistry, 2008;23(1):33- 37.

13. Ojha SK, Nandave M, Arora S, Narang R, Dinda AK, Arya DS. Chronic administration of Tribulus terrestris Linn. Extract improves cardiac function and attenuates myocardial infarction in rats. Int J Pharmacol, 2008;4:1-10.

14. Tasf, Hansel, H., Belce, A., Ilvan, S., Argon, A., Camlica, H., Topuz, E., 'oxidative stress in ovarian cancer', Medical Oncology, 2005;22(1):11-15. 